\title{
Avaliação da satisfação profissional dos enfermeiros por áreas de prestação de cuidados*
}

\author{
Nurses' job satisfaction assessment by areas of care \\ Evaluación de la satisfacción laboral del enfermero por áreas de prestación de cuidados
}

Como citar este artigo:

Silva CINP, Potra TMFS. Nurses' job satisfaction assessment by areas of care. Rev Esc Enferm USP. 2021;55:e03730. https://doi.org/10.1590/S1980220X2020013203730

\author{
Cláudia Isabel Neves Pacheco \\ da Silva ${ }^{1}$ \\ Deresa Maria Ferreira dos Santos \\ Potra $^{2}$ \\ * Extraído da dissertação: "Satisfação Profissional \\ dos Enfermeiros numa Unidade Local de Saúde”, \\ Escola Superior de Enfermagem de Lisboa, 2017. \\ ${ }^{1}$ Unidade Local de Saúde do Litoral \\ Alentejano, Serviço de Urgência Básico de \\ Odemira, Santiago do Cacém, Portugal. \\ ${ }^{2}$ Escola Superior de Enfermagem \\ de Lisboa, Lisboa, Portugal.
}

\begin{abstract}
Objective: To analyze the level of nurses' job satisfaction according to the area of care provided in a Local Health Unit, in Portugal. Method: This is a quantitative, descriptive, analytical study, with observational and cross-sectional design. The Job Satisfaction Assessment Instrument was used for data collection. Results: Sample $(\mathrm{n}=218)$, predominantly female $(84.6 \%)$, with a mean age of 33 years. The area of care delivery influences total job satisfaction $(p<0.05)$ and some of the dimensions studied $(p<0.05)$. Nurses from Community Care Units show greater job satisfaction, followed by nurses from Indirect Care. Critical Care nurses show lower job satisfaction, followed by those who work in outpatient's units. Most nurses show lower satisfaction level in the aspect Management Bodies and in the dimension Quality of Care Delivery. Conclusion: The most satisfied nurses are those from the Community Care Units and the nurses with the lowest level of job satisfaction are those from Critical Care.
\end{abstract}

\section{DESCRIPTORS}

Job Satisfaction; Nurses; Delivery of Health Care. 


\section{INTRODUÇÃO}

A satisfação profissional (SP) é uma variável do comportamento organizacional crucial para melhorar o funcionamento das organizações, constituindo um potente indicador de gestão, pelo impacto que pode ter no desempenho. Neste sentido, para que se possam definir estruturas de suporte e políticas organizacionais que promovam esta variável, é imperativo que a conheçamos ${ }^{(1)}$. Os profissionais que se sentem valorizados na sua profissão apresentam menor probabilidade de abandonar o trabalho, mais comportamentos de cidadania organizacional, maior satisfação, melhor desempenho, mais confiança, desenvolvem sentimentos de lealdade para com a organização e menor absentismo voluntário. A $\mathrm{SP}$ relaciona-se diretamente com a saúde mental, sendo que uma maior satisfação reduz a incidência de burnout e, consequentemente, aumenta a qualidade dos cuidados ${ }^{(2-3)}$.

A retenção de enfermeiros satisfeitos é essencial para garantir a elevada qualidade dos cuidados, sendo que nesta área os líderes autênticos desempenham um papel importante na criação de ambientes com um suporte efetivo das práticas profissionais, resultando numa percepção de elevada Qualidade da Prestação de Cuidados (QPC) e maior SP ${ }^{(4)}$. As crescentes necessidades de qualidade da população exigem uma inovação na prestação de cuidados, que deve ser sustentada pela investigação. Apesar de a gestão de talentos poder constituir um desafio para a gestão de recursos humanos, esta não deve ser encarada como uma dificuldade, mas antes como uma oportunidade para responder às referidas necessidades, elevando os padrões de qualidade e acrescentando valor às organizações.

As unidades locais de saúde são modelos organizacionais que têm como objetivo melhorar a capacidade e otimização dos cuidados prestados à população, por meio de uma gestão integrada de unidades de diferentes áreas, retratando, talvez, o expoente máximo da integração e continuidade de cuidados. Este tipo de organizações procura "melhorar a capacidade de resposta do sistema da saúde e otimizar a resposta dos serviços por meio de uma gestão integrada das várias unidades de saúde de uma região"(5). Representa uma integração vertical de cuidados de saúde, na medida em que é a única entidade gestora que detém o controle de duas (ou mais) organizações, sendo que pelo menos uma delas utiliza como input o output da outra ${ }^{(6)}$. Assim, numa mesma organização existem diferentes áreas de cuidados: cuidados de saúde primários (CSP), cuidados hospitalares $(\mathrm{CH})$ e cuidados continuados (CC).

Uma Unidade Local de Saúde (ULS) no sul de Portugal integra as seguintes unidades distribuídas pelas três áreas de cuidados: a) Cuidados de Saúde Primários: unidades de cuidados na comunidade (UCC), unidades de cuidados de saúde personalizados (UCSP) e unidades de saúde pública (USP); b) Cuidados Hospitalares: Internamento (Medicina Interna, Cirurgia e Ortopedia); Ambulatório (Hospital dia, Consulta externa, Cirurgia de ambulatório, Técnicas de gastrenterologia); Cuidados Críticos (Unidade de cuidados intensivos, Bloco cirúrgico e Serviços de urgência); Cuidados Indiretos (Esterilização, Comissões técnicas e Formação); e os Cuidados Continuados (unidade de convalescença e equipe de gestão de altas).

Com base na diversidade de contextos de cuidados, considerou-se que a SP dos enfermeiros numa ULS poderia variar de acordo com a áreas de cuidados. Assim, em face ao exposto, a questão de investigação estabelecida inicialmente, e que orientou todo o processo de investigação foi: a satisfação profissional dos enfermeiros é influenciada pela área de cuidados?

O presente estudo teve como objetivo geral analisar o grau de satisfação profissional dos enfermeiros segundo a área de cuidados numa ULS, em Portugal. Em termos de objetivos específicos, pretende-se:

a) comparar o grau de satisfação profissional total entre as diversas áreas de cuidados;

b) comparar o grau de satisfação profissional por dimensão entre as diversas áreas de cuidados;

c) conhecer o grau de satisfação por área de cuidados.

\section{MÉTODO}

\section{Desenho do estudo}

Estudo descritivo, quantitativo, com desenho observacional e transversal.

\section{Cenário}

Foi realizado numa Unidade Local de Saúde do sul de Portugal. A população do estudo correspondia a todos os enfermeiros da ULS em funções no período de 9 a 12 de janeiro de 2017, 311 enfermeiros, aos quais foi entregue um questionário. Os critérios de inclusão foram enfermeiros no exercício efetivo com questionário preenchido, tendo a amostra final sido constituída por 218 enfermeiros.

\section{Coleta de Dados}

Foi aplicado o questionário Instrumento de Avaliação da Satisfação Profissional (IASP), desenvolvido pelo Centro de Estudos e Investigação em Saúde da Universidade de Coimbra (Ceisuc), Portugal, por se adaptar às especificidades dos profissionais de saúde, e por conter dimensões da qualidade orientadas para as questões do local de trabalho, da prestação de cuidados e da melhoria contínua. Este encontra-se dividido em 6 grupos. No primeiro grupo é realizada a identificação da unidade. $\mathrm{O}$ segundo, terceiro e quarto grupos do instrumento são compostos pelas escalas "Qualidade do Local de Trabalho" (QLT); "Qualidade na Prestação de Cuidados" (QPC) e "Melhoria Contínua da Qualidade" (MCQ). Na QLT pretende-se avaliar a satisfação dos enfermeiros face às políticas de recursos humanos, designadamente, no que se refere aos recursos humanos propriamente ditos e aos órgãos de gestão. É também avaliada a satisfação face à segurança e organização do serviço onde trabalham e ao vencimento auferido, tendo em conta o seu desempenho, experiência e responsabilidade. A QLT contempla ainda uma faceta referente à moral, em que se avalia a satisfação em relação ao seu superior hierárquico direto (coordenador), bem como em face ao seu estado 
de espírito. A escala da QPC avalia a satisfação no que se refere aos cuidados diretos, à capacidade e sensibilidade dos profissionais, à organização dos cuidados, à informação prestada aos clientes, bem como em face às instalações dos serviços e o custo dos cuidados. Por fim, a MCQ refere-se ao investimento na qualidade dos cuidados, avaliando a satisfação relativamente ao ambiente de trabalho, partilha de ideias, conhecimentos e expectativas de qualidade dos profissionais, bem como preocupação em como se investe para fazer bem à primeira vez e com as medidas implementadas para diminuir o desperdício.

As respostas foram dadas numa escala de Likert de 5 pontos, em que: 1 significa "Mau"; 2 "Regular"; 3 "Bom"; 4 "Muito Bom"; 5 "Excelente", estando prevista também a opção "não se aplica". A cada item da escala de Likert (1 a 5 pontos) corresponde uma escala percentual de $0 \%$ a $100 \%$, de acordo com o seguinte critério: mau - 0\%; regular - 50\%; bom - 70\%; muito bom - 90\%; excelente - 100\%. $\mathrm{Na}$ tentativa de compreender melhor o fenômeno, foi incluída uma questão (grupo cinco) que pedia aos participantes que classificassem o seu grau de satisfação global na instituição, tendo sido utilizada uma escala tipo Likert, de 1 a 4, com inversão na pontuação atribuída (1 representa a situação mais satisfatória, e 4 a menos satisfatória). Por fim, o grupo seis incluía questões para a caraterização da amostra (gênero; idade; situação familiar; agregado familiar; número de filhos; rendimento familiar; formação acadêmica; categoria profissional; e emigração), cujas respostas eram facultativas.

O IASP apresentou uma excelente fiabilidade em estudos anteriores, dado que na análise do Alpha de Cronbach os resultados obtidos situam-se acima de 0,706 e na maioria das situações, acima de 0,9 . Foi calculada a consistência interna neste estudo, tendo-se obtido valores de Alpha de Cronbach superiores a 0,889 e a 0,9 em todas as escalas, subescalas e facetas. Assim, a consistência interna classifica-se como "boa" e "muito boa".

\section{ANÁLISE E TRATAMENTO DOS DADOS}

A organização, sistematização e o tratamento estatístico dos dados foram realizados com recurso ao programa SPSS 24.0 (Statistical Package for the Social Sciences), possibilitando sua análise de forma descritiva, verificação da existência de relações entre as variáveis, e comparação entre grupos, de modo a determinar se as diferenças entre eles são estatisticamente significativas ${ }^{(7)}$. Foram utilizadas medidas estatísticas descritivas e de comparação para analisar a SP dos enfermeiros na ULS. Como estatística descritiva foi utilizada a média, desvio padrão, frequências absolutas e relativas. Para determinar a diferença de médias nas dimensões da satisfação profissional segundo fatores (sociodemográficos e organizacionais) foram utilizados os testes paramétricos t-Student e One-Way ANOVA, pois, de acordo com especialistas $^{(8)}$, para amostras grandes $(\mathrm{n}=218)$ estes são bastante robustos mesmo quando a distribuição da variável sob o estudo não é do tipo normal. O teste Post Hoc de Sheffe foi utilizado para determinar em que grupos ocorrem as diferenças estatisticamente significativas. Foi considerado o nível de significância $p=0,05$, com um intervalo de confiança de $95 \%$.
Ou seja, para < 0,05 admite-se a existência de diferença ou associação entre grupos. Se > 0,05 não se admite a existência de diferença ou associação entre grupos.

\section{Aspectos ÉTICOS}

Foram considerados os procedimentos éticos (consentimento informado, confidencialidade, privacidade dos dados e instruções) durante a elaboração do questionário, nos procedimentos para a sua aplicação e tratamento de dados, no que se refere à organização e aos participantes no estudo. A aplicação do IASP foi autorizada pelo autor do instrumento de coleta de dados. Mediante o parecer positivo da Comissão de Ética, a aplicação dos questionários foi autorizada pelo Conselho de Administração da ULS. A participação no estudo foi voluntária, estando todos os questionários acompanhados de uma carta de apresentação dos investigadores onde foram explicados os objetivos do estudo. Com vista a preservar a confidencialidade das respostas, os questionários foram distribuídos ainda com um envelope que possibilitava seu fechamento. Os próprios enfermeiros colocaram o referido envelope numa urna, também fechada.

\section{RESULTADOS}

Os 218 participantes que constituem a amostra traduzem uma taxa de resposta de 70,1\%. A amostra é majoritariamente do sexo feminino (84,7\%), com a média etária de 33 anos, com um desvio padrão de 13 anos. A maioria (64,0\%) é casada ou vive em união estável, com um agregado composto por 3 a 4 elementos (56,0\%), tem 1 a 2 filhos (59,0\%). Relativamente aos aspectos organizacionais, cerca de metade tem a categoria de enfermeiro, grau de licenciado e realiza em média 35 horas de cuidados por semana $(58,0 \%, 54,0 \%$ e $50,0 \%$, respetivamente). A outra metade distribui-se entre as restantes categorias e graus, sendo as com maior representatividade a categoria de especialista, o grau de pós-graduação e que realizam entre 36 a $40 \mathrm{~h}$ de cuidados por semana $(22,0 \%$, $23,0 \%$ e 40,0\%). A grande maioria (81,3\%) realiza trabalho em regime de acumulação de funções. Por fim, em relação ao tempo de serviço, a amostra apresenta maior porção no intervalo (46,7\%) 0 - 10 anos, comparativamente aos restantes, 11 a 20 anos (29\%) e 21 a 30 anos (19,3\%). A maioria dos participantes trabalha nos Cuidados Hospitalares $(\mathrm{CH})$ (57,8\%), seguidos dos Cuidados de Saúde Primários (CSP) (39,9\%) e dos Cuidados Continuados (CC) (2,3\%). Dada a diversidade de contextos que integram a área dos $\mathrm{CH}$, foram criados 4 grupos tendo em consideração as semelhanças entre a prestação de cuidados: Cuidados Críticos (Bloco Cirúrgico, Intensivos, Urgência), em que o cliente necessita de cuidados $24 \mathrm{~h} /$ dia e encontra-se numa situação de instabilidade hemodinâmica; no Internamento (Medicina Interna, Ortopedia, Cirurgia), onde o cliente necessita de cuidados $24 \mathrm{~h} /$ dia e a priori apresenta maior estabilidade, encontrando-se em fase de compensação; Ambulatório (Hospital dia, Consulta Externa, Cirurgia de Ambulatório, Técnicas de Gastroenterologia), onde por norma os cuidados são prestados de forma programada e não necessitam da presença de enfermeiros nas 24h; e Indiretos (Comissões, Esterilização e 
Formação), sendo prestados apenas no período diurno e de forma programada, sem contato direto com o cliente. Os CSP incluem 5 Unidades de Cuidados de Saúde Personalizados e 5 Unidades de Cuidados na Comunidade. Os Cuidados Continuados incluem uma Unidade de Convalescença e uma Equipe de Gestão de Altas.
$\mathrm{Na}$ análise da média total de SP por área de cuidados verificou-se que a maioria dos enfermeiros das várias áreas de cuidados (cinco) apresenta uma satisfação classificada de "Regular" (58,4\% a 67,8\%), havendo um contexto com a classificação de "Bom" (UCC) e outro com "Muito Bom" (Indiretos) (Tabela 1).

Tabela 1 - Satisfação Profissional total de enfermeiros por área de cuidados - Sul de Portugal, 2017.

\begin{tabular}{|c|c|c|c|c|c|c|c|c|c|c|c|c|}
\hline \multirow{5}{*}{$\begin{array}{l}\text { Média Total } \\
\text { de SP } \\
\text { (QLT, QPC, } \\
\text { MCQ) }\end{array}$} & & $\begin{array}{l}\text { INT } \\
(1)\end{array}$ & $\begin{array}{c}\text { AMB } \\
(2)\end{array}$ & $\begin{array}{c}\mathrm{CCr} \\
(3)\end{array}$ & $\begin{array}{l}\text { IND } \\
(4)\end{array}$ & $\begin{array}{c}\text { UCC } \\
(5)\end{array}$ & $\begin{array}{c}\text { UCSP/ } \\
\text { USP } \\
(6)\end{array}$ & $\begin{array}{c}\text { UC/EGA } \\
(7)\end{array}$ & $\Sigma$ & $\mathbf{F}$ & $p$ & Post Hoc \\
\hline & & 47 & 11 & 56 & 3 & 22 & 63 & 5 & 207 & & & \\
\hline & $M$ (arit.) & 2,89 & 2,42 & 2,57 & 4,57 & 3,34 & 2,84 & 2,77 & 2,83 & \multirow{3}{*}{9,52} & \multirow{3}{*}{$0,000^{*}$} & \multirow{3}{*}{$\begin{array}{c}4>1,2,3,6,7 \\
5>2,3\end{array}$} \\
\hline & $M(\%)$ & 67,80 & 58,40 & 61,40 & 95,70 & 76,80 & 68,80 & 65,40 & 66,60 & & & \\
\hline & DP & 0,72 & 0,64 & 0,57 & 0,67 & 0,52 & 0,54 & 0,55 & 0,67 & & & \\
\hline
\end{tabular}

AMB: Ambulatório; CCr: Cuidados Críticos; IND: Cuidados Indiretos; INT: Internamento; DP: desvio padrão; EGA: Equipe de gestão de altas; F: One-Way ANOVA; M (arit.): Média aritmética; M (\%): Média em percentagem; MCQ: Melhoria continua da qualidade; QLT: Qualidade do local de trabalho; QPC: Qualidade da prestação de cuidados; UC: Unidade de convalescença; UCC: Unidade de cuidados na comunidade; UCSP: Unidade de cuidados de saúde personalizados; USP: Unidade de saúde pública ${ }^{*} p<0,005$

Existem diferenças estatisticamente significativas segundo a área de prestação de cuidados: os enfermeiros nos Cuidados Indiretos apresentam maior satisfação (95,7\%), comparativamente aos contextos: Internamento, Ambulatório e Cuidados Críticos, UCC, UCSP/USP e UC/EGA (valores variam entre 58,4\% e 76,8\%); os enfermeiros das UCC $(76,8 \%)$ apresentam maior satisfação que os do Ambulatório (58,4\%) e Cuidados Críticos $(61,4 \%)$.

Verificaram-se diferenças estatisticamente significativas $(p<0,05)$ na análise por área de cuidados em todas as dimensões da satisfação profissional: "QLT" $(p=0,000)$; "política de recursos humanos" ( $p=0,001)$; "órgãos de gestão" ( $p=0,000)$; "recursos humanos" $(\mathrm{P}=0,035)$; "moral" $(p=0,000)$; "coordenador" $(p=0,000)$; "estado de espírito" $(p=0,001)$; "QPC" $(p=0,000)$; e "MCQ" $(p=0,000)$. Apenas não se identificaram diferenças estatisticamente significativas quanto aos "recursos tecnológicos financeiros" ("vencimento" e "local de trabalho e equipamento") (Tabela 2).

Tabela 2 - Satisfação profissional de enfermeiros segundo a área de cuidados - Sul de Portugal, 2017.

\begin{tabular}{|c|c|c|c|c|c|c|c|c|c|c|c|c|c|c|}
\hline \multirow[b]{2}{*}{ Escala } & \multirow[b]{2}{*}{ Sub escala } & \multirow[b]{2}{*}{ Faceta } & & \multirow[b]{2}{*}{$\Sigma$} & \multicolumn{4}{|c|}{$\mathrm{CH}$} & \multicolumn{2}{|c|}{ CSP } & \multirow{2}{*}{$\begin{array}{c}\text { CC } \\
\text { UC/ } \\
\text { EGA } \\
(7) \\
\end{array}$} & \multirow[b]{2}{*}{$\mathbf{F}$} & \multirow[b]{2}{*}{$\mathbf{p}$} & \multirow[b]{2}{*}{ Post Hoc } \\
\hline & & & & & $\begin{array}{l}\text { INT. } \\
(1)\end{array}$ & $\begin{array}{c}\text { AMB. } \\
\text { (2) }\end{array}$ & $\begin{array}{l}\mathrm{CCr} \\
(3)\end{array}$ & $\begin{array}{c}\text { IND } \\
(4)\end{array}$ & $\begin{array}{c}\text { UCC } \\
\text { (5) }\end{array}$ & $\begin{array}{c}\text { UCSP/ } \\
\text { USP } \\
(6)\end{array}$ & & & & \\
\hline & & & $\mathbf{n}$ & 217 & 49 & 13 & 59 & 4 & 22 & 65 & 5 & & & \\
\hline \multirow{3}{*}{\multicolumn{3}{|c|}{ QUALIDADE DO LOCAL DE TRABALHO }} & $M(\%)$ & 61,86 & 62,27 & 57,99 & 56,74 & 75,98 & 71,69 & 63,07 & 60,34 & & & \\
\hline & & & $M$ (arit.) & 2,59 & 2,61 & 2,40 & 2,34 & 3,30 & 3,08 & 2,65 & 2,52 & 4,586 & $0,000^{*}$ & $5>3$ \\
\hline & & & DP & 0,68 & 0,78 & 0,82 & 0,51 & 0,87 & 0,59 & 0,63 & 0,45 & & & \\
\hline \multirow{3}{*}{\multicolumn{3}{|c|}{ Política de Recursos Humanos }} & $M(\%)$ & 55,86 & 54,49 & 53,25 & 51,85 & 70,00 & 66,16 & 56,94 & 55,33 & & & \\
\hline & & & $M$ (arit.) & 2,29 & 2,22 & 2,16 & 2,09 & 3,00 & 2,81 & 2,35 & 2,27 & 4,174 & $0,001^{*}$ & $5>3$ \\
\hline & & & DP & 0,67 & 0,70 & 0,74 & 0,59 & 0,80 & 0,65 & 0,63 & 0,52 & & & \\
\hline \multirow{3}{*}{\multicolumn{3}{|c|}{ Orgãos de Gestão }} & $M(\%)$ & 51,64 & 48,21 & 48,08 & 41,95 & 60,00 & 68,41 & 51,77 & 56,00 & & & \\
\hline & & & M (arit.) & 2,08 & 1,96 & 1,96 & 1,84 & 2,50 & 2,92 & 2,09 & 2,30 & 5,878 & $0,000^{*}$ & $5>1,2,3,6$ \\
\hline & & & DP & 0,82 & 0,90 & 0,78 & 0,64 & 1,40 & 0,81 & 0,69 & 0,89 & & & \\
\hline \multirow{3}{*}{\multicolumn{3}{|c|}{ Recursos Humanos }} & $M(\%)$ & 59,25 & 58,42 & 56,46 & 55,90 & 80,67 & 64,36 & 61,08 & 54,80 & \multirow{3}{*}{2,315} & \multirow{3}{*}{$0,035^{*}$} & \\
\hline & & & $M$ (arit.) & 2,46 & 2,42 & 2,32 & 2,29 & 3,53 & 2,72 & 2,55 & 2,24 & & & \\
\hline & & & $\mathrm{DP}$ & 0,76 & 0,69 & 0,87 & 0,76 & 1,14 & 0,72 & 0,75 & 0,43 & & & \\
\hline
\end{tabular}




\begin{tabular}{|c|c|c|c|c|c|c|c|c|c|c|c|c|}
\hline \multirow[b]{2}{*}{ Sub escala } & & \multirow[b]{2}{*}{$\Sigma$} & \multicolumn{4}{|c|}{$\mathrm{CH}$} & \multicolumn{2}{|c|}{ CSP } & \multirow{2}{*}{$\begin{array}{c}\mathrm{CC} \\
\mathrm{UC} \\
\mathrm{EGA} \\
(7)\end{array}$} & \multirow[b]{2}{*}{$\mathbf{F}$} & \multirow[b]{2}{*}{$\mathbf{p}$} & \multirow[b]{2}{*}{ Post Hoc } \\
\hline & & & $\begin{array}{l}\text { INT. } \\
\text { (1) }\end{array}$ & $\begin{array}{c}\text { AMB. } \\
(2)\end{array}$ & $\begin{array}{c}\mathrm{CCr} \\
(3)\end{array}$ & $\begin{array}{l}\text { IND } \\
(4)\end{array}$ & $\begin{array}{c}\text { UCC } \\
(5)\end{array}$ & $\begin{array}{c}\text { UCSP/ } \\
\text { USP } \\
(6)\end{array}$ & & & & \\
\hline \multirow[t]{3}{*}{ Moral } & $M(\%)$ & 71,03 & 70,16 & 64,49 & 62,83 & 89,23 & 86,15 & 74,18 & 70,62 & \multirow{3}{*}{5,462} & \multirow{3}{*}{$0,000^{*}$} & \multirow{3}{*}{$5>3$} \\
\hline & $M$ (arit.) & 3,05 & 3,01 & 2,72 & 2,64 & 3,96 & 3,81 & 3,21 & 3,03 & & & \\
\hline & DP & 0,99 & 1,12 & 1,26 & 0,73 & 1,26 & 0,89 & 0,90 & 0,65 & & & \\
\hline \multirow[t]{3}{*}{ Coordenador } & $M(\%)$ & 71,49 & 69,29 & 65,10 & 62,91 & 91,14 & 87,77 & 75,43 & 72,18 & \multirow{3}{*}{5,348} & \multirow{3}{*}{$0,000^{*}$} & \\
\hline & $M$ (arit.) & 3,07 & 2,96 & 2,76 & 2,65 & 4,11 & 3,89 & 3,27 & 3,11 & & & \\
\hline & DP & 1,09 & 1,23 & 1,23 & 0,84 & 1,43 & 1,02 & 0,99 & 0,74 & & & \\
\hline \multirow[t]{3}{*}{ Estado de Espírito } & $M(\%)$ & 68,52 & 74,90 & 64,17 & 62,37 & 72,50 & 77,27 & 67,38 & 62,00 & \multirow{3}{*}{4,203} & \multirow{3}{*}{$0,001^{*}$} & \multirow{3}{*}{$1,5>3$} \\
\hline & $M$ (arit.) & 2,93 & 3,24 & 2,71 & 2,62 & 3,13 & 3,36 & 2,87 & 2,60 & & & \\
\hline & DP & 0,84 & 0,87 & 1,27 & 0,72 & 0,85 & 0,80 & 0,74 & 0,42 & & & \\
\hline \multirow[t]{3}{*}{ Recursos Tecnológicos e Financeiros } & $M(\%)$ & 52,53 & 56,88 & 52,42 & 51,74 & 53,57 & 51,95 & 50,48 & 44,29 & \multirow{3}{*}{1,364} & \multirow{3}{*}{0,231} & \\
\hline & $M$ (arit.) & 2,13 & 2,34 & 2,12 & 2,09 & 2,18 & 2,10 & 2,02 & 1,89 & & & \\
\hline & DP & 0,65 & 0,75 & 0,54 & 0,69 & 0,51 & 0,59 & 0,57 & 0,41 & & & \\
\hline \multirow[t]{3}{*}{ Vencimento } & $M(\%)$ & 22,12 & 32,31 & 17,95 & 17,23 & 25,00 & 25,00 & 18,97 & 16,67 & \multirow{3}{*}{1,144} & \multirow{3}{*}{0,338} & \\
\hline & $M$ (arit.) & 1,44 & 1,65 & 1,36 & 1,34 & 1,50 & 1,50 & 1,38 & 1,33 & & & \\
\hline & DP & 0,67 & 0,76 & 0,63 & 0,68 & 0,43 & 0,75 & 0,59 & 0,33 & & & \\
\hline \multirow[t]{3}{*}{ Local de trabalho e equipamento } & $M(\%)$ & 62,79 & 67,35 & 63,85 & 62,88 & 63,75 & 60,91 & 60,15 & 56,00 & \multirow{3}{*}{0,947} & \multirow{3}{*}{0,462} & \\
\hline & $M$ (arit.) & 2,64 & 2,87 & 2,69 & 2,64 & 2,69 & 2,55 & 2,51 & 2,30 & & & \\
\hline & DP & 0,89 & 0,95 & 0,89 & 0,95 & 0,69 & 0,71 & 0,85 & 0,67 & & & \\
\hline \multirow[t]{3}{*}{ QUALIDADE DA PRESTAÇÃO DE CUIDADOS } & $M(\%)$ & 67,87 & 69,52 & 63,33 & 64,19 & 96,79 & 76,88 & 65,45 & 67,71 & \multirow{3}{*}{6,944} & & \\
\hline & $M$ (arit.) & 2,89 & 2,98 & 2,67 & 2,71 & 4,68 & 3,34 & 2,77 & 2,89 & & $0,000^{*}$ & $\begin{array}{l}4>1,2,3 \\
6,7: 5>3\end{array}$ \\
\hline & DP & 0,77 & 0,76 & 0,72 & 0,72 & 1,89 & 0,59 & 0,59 & 0,65 & & & \\
\hline MELHORIA CONTINUA DA QUALIDADE & $M(\%)$ & 70,40 & 70,87 & 61,54 & 63,64 & 94,06 & 81,59 & 72,42 & 68,00 & & & \\
\hline & $M$ (arit.) & 3,02 & 3,04 & 2,58 & 2,68 & 4,41 & 3,58 & 3,12 & 2,90 & 6,944 & $0,000^{*}$ & $4,5>2,3$ \\
\hline & $\mathrm{DP}$ & 0,83 & 0,88 & 0,85 & 0,74 & 1,26 & 0,61 & 0,68 & 0,85 & & & \\
\hline
\end{tabular}

AMB: Ambulatório; CC: Cuidados Continuados; CCr: Cuidados Críticos; $\mathrm{CH}$ : Cuidados Hospitalares; CSP: Cuidados de saúde primários; IND: Cuidados Indiretos; INT: Internamento; DP: desvio padrão; EGA: Equipe de gestão de altas; F: One-Way ANOVA; M (arit.): Média aritmética; $M(\%)$ : Média em percentagem; MCQ: Melhoria continua da qualidade; QLT: Qualidade do local de trabalho; QPC: Qualidade da prestação de cuidados; UC: Unidade de convalescença; UCC: Unidade de cuidados na comunidade; UCSP: Unidade de cuidados de saúde personalizados; USP: Unidade de saúde pública ${ }^{*} p<0,005$

Na escala "QLT" e subescala "política de recursos humanos", o nível de satisfação profissional dos enfermeiros das UCC apresenta maior satisfação $(71,69 \%$ e $66,16 \%)$ que o grupo de Cuidados Críticos $(56,74 \%$ e $51,85 \%)$. Relativamente aos "órgãos de gestão", os enfermeiros das UCC apresentam maior satisfação $(68,41 \%)$ do que os grupos das UCSP/USP (51,77\%), dos de Internamento (48,21\%), de Ambulatório (48,08\%), e de Críticos (41,95\%).

No tocante à faceta "recursos humanos" e "coordenador", apesar da diferença estatisticamente significativa entre os grupos, a utilização do teste Post-Hoc de Sheffe não possibilitou identificar qual o que tem maior nível de satisfação. Contudo, verifica-se uma tendência de seguimento do padrão identificado nas restantes facetas/dimensões, em que os enfermeiros do grupo de Cuidados Indiretos tendem a exibir maior satisfação $(80,67 \%$ e $91,14 \%)$ do que os enfermeiros de Cuidados Críticos (55,9\% e 62,91\%). Relativamente à subescala "moral" e "estado de espírito" os enfermeiros das UCC exibem maior satisfação $(86,15 \%$ e $77,27 \%)$ que o grupo integrante da área de Cuidados Críticos $(62,83 \% \mathrm{e}$ $62,37 \%$ ). Surge ainda como grupo com maior satisfação em face ao estado de espírito, o que diz respeito às áreas de Internamento (Tabela 2).

Relativamente à "QPC", os enfermeiros de Cuidados Indiretos apresentam maior nível de satisfação profissional, com 96,79\%, do que os enfermeiros dos grupos de Internamento $(69,52 \%)$ e de Ambulatório $(63,33 \%)$, de Cuidados Críticos (64,19\%), das UCSP/USP (65,45\%), e da UC/EGA (67,71\%). Ainda nesta dimensão foi também identificada uma diferença estatisticamente significativa entre o grupo das UCC e os de Cuidados Críticos, sendo que o primeiro apresenta maior satisfação $(76,88 \%)$ que o segundo $(64,19 \%)$. 
No que se refere a "MCQ", tanto os enfermeiros integrantes nas UCC como dos Cuidados Indiretos apresentam maior valor de satisfação $(81,59 \%$ e $94,06 \%$, respetivamente) que o grupo de Ambulatório (61,54\%) e de Cuidados Críticos (63,64\%).

Os participantes definiram o grau de satisfação na sua instituição (qual é o seu grau de satisfação nesta instituição?), tendo em conta as hipóteses: 1 - "muito insatisfeito/a",
2 - "insatisfeito/a", 3 - "nem satisfeito/a nem insatisfeito/a", 4 - "satisfeito", e 5 - "muito satisfeito/a". Constataram-se diferenças estatisticamente significativas $(p=0,000)$ no grau de satisfação global segundo a área de prestação de cuidados.

Os enfermeiros da área de cuidados da UCC percebem-se num grau de satisfação superior, comparativamente aos enfermeiros de Ambulatório e Cuidados Críticos.

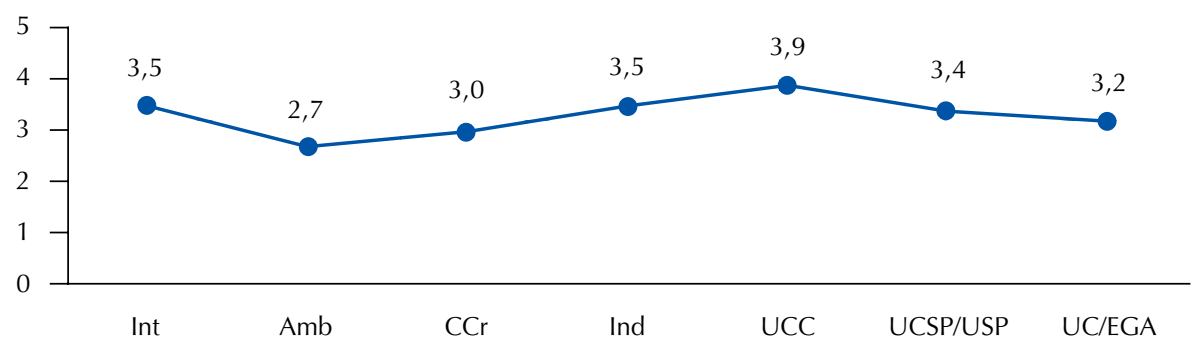

1: Muito Insatisfeito; 2: Insatisfeito; 3: Nem Satisfeito/Nem Insatisfeito; 4: Satisfeito; 5: Muito Satisfeito; Int: Internamento; Amb: Ambulatório; CCr: Cuidados Críticos; Ind: Cuidados Indiretos; UCC: Unidade de Cuidados na Comunidade; UCSP: Unidade de Cuidados de Saúde Personalizados; USP: Unidade de Saúde Pública; UC/EGA: Unidade de Convalescença/Equipe de gestão de altas

Figura 1 - Grau de satisfação de enfermeiros por área de cuidados - Sul de Portugal, 2017.

A maioria dos profissionais posiciona-se num ponto de neutralidade ("nem satisfeitos/nem insatisfeitos"). Destaca-se o grupo de profissionais pertencentes às UCC que se posiciona mais próximo do grau de satisfação designado por "satisfeito". Os grupos com menor classificação são os que integram os Cuidados de Ambulatório e os Cuidados Críticos, que se consideram, respetivamente, "insatisfeitos" e "nem satisfeitos/nem insatisfeitos" (Figura 1).

\section{DISCUSSÃO}

A área de cuidados influencia a satisfação profissional dos enfermeiros, total e por dimensão (QLT, QPC e MCQ), bem como a sua percepção do nível de satisfação, sendo congruente com os estudos anteriores ${ }^{(9-10)}$ que concluíram a existência de relação entre a satisfação e o contexto de exercício de funções.

\section{Satisfação Profissional.}

Os enfermeiros com maior SP total exercem funções nas unidades de Cuidados Indiretos e nas UCC. As funções inerentes aos cuidados Indiretos coadunam-se com uma intervenção autônoma em face ao planejamento e implementação dos cuidados, característica esta também inerente ao modelo organizacional das UCC, pelo que se coloca a hipótese de ser este um dos motivos para a maior satisfação identificada em ambos os contextos, dado que a autonomia e a participação na tomada de decisão são os fatores que mais contribuem para a promoção desta dimensão do comportamento organizacional ${ }^{(11-12)}$. Um suporte que promova a autonomia e independência na organização dos cuidados é um fator promotor da satisfação profissional para os enfermeiros desta área de cuidados, contribuindo para que se encontrem mais satisfeitos em face às recompensas intrínsecas como a natureza do trabalho, alcance de objetivos e desenvolvimento pessoal ${ }^{(13-14)}$.

\section{Satisfação Profissional por dimensão}

A análise da SP por dimensão possibilitou ainda identificar que os enfermeiros menos satisfeitos são os que trabalham em ambientes de Cuidados Críticos. Os enfermeiros de Cuidados Críticos são o grupo que apresenta menor satisfação em face à qualidade do local de trabalho, isto é, às políticas de recursos humanos (órgãos de gestão e recursos humanos) e moral (coordenação e estado de espírito), à qualidade da prestação de cuidados e melhoria contínua da qualidade.

A relação estabelecida com os órgãos de gestão é determinante para a satisfação profissional dos enfermeiros, sendo estes os responsáveis pelas políticas organizacionais que irão modelar as estratégias de suporte para todos os profissionais, nomeadamente no que se refere aos recursos humanos (proporção, número, continuidade). A forma como os órgãos de gestão comunicam-se com, tratam, apoiam e reconhecem os profissionais são fatores identificados como fontes da satisfação dos enfermeiros de cuidados de saúde primários.

Especificamente neste estudo, foi identificado um grupo que incorpora esta área de cuidados, os enfermeiros das UCC, cujo modelo organizacional possibilita um canal de comunicação direto com os órgãos de gestão em virtude de ser a única unidade coordenada por enfermeiros ${ }^{(13-14)}$. Outro estudo ${ }^{(15)}$ corrobora este resultado, tendo identificado a UCC como correspondendo à unidade de cuidados de saúde primários com enfermeiros mais satisfeitos em face aos órgãos de gestão. A liderança baseada na motivação, suporte e comunicação efetiva (transformacional), em detrimento do culto do controle e punição (transacional), é promotora 
da satisfação profissional. Os enfermeiros sentem-se mais satisfeitos em face a uma liderança baseada num modelo de gestão bottom-up em detrimento de um top-down $n^{(16-17)}$.

O presente estudo identificou também os enfermeiros de Cuidados Críticos como grupo de menor satisfação com a qualidade do local de trabalho. No que se refere aos órgãos de gestão, os enfermeiros de cuidados críticos aparentam almejar uma liderança que promova a comunicação bidirecional, o reconhecimento e a transparência dos processos, sendo que nesta área de cuidados a liderança do tipo transacional é fonte de insatisfação profissional ${ }^{(14,16)}$. Foi ainda verificado que a menor satisfação com os órgãos de gestão é transversal à maioria dos enfermeiros (Internamento, Ambulatório e das UCSP/USP), que sentem necessidade de maior proximidade com as chefias de topo, na medida em que as formas de liderança que suportam a construção de confiança, como a comunicação e respeito pelas opiniões dos funcionários, são promotoras da satisfação profissional ${ }^{(18)}$. Na mesma linha de pensamento, surge a satisfação com a liderança das chefias intermédias, neste estudo designadas por coordenação.

Não foi possível estatisticamente identificar quais os grupos com maiores diferenças na satisfação, contudo os resultados apontam a tendência de que os enfermeiros com maior satisfação serão os das UCC e os com menor, os de Cuidados Críticos. Coloca-se a hipótese de o fato de o coordenador direto de enfermagem das UCC ser coincidente com o coordenador da unidade poder contribuir para a maior satisfação deste grupo em face aos restantes. Esta posição constitui-se como fator promotor de uma comunicação eficaz e fortalecimento de relações interpessoais com a equipe, uma vez que a tomada de decisão é baseada no conhecimento real e contextualizado nas dificuldades e oportunidades do contexto de prática dos enfermeiros.

Ainda no que se refere à Qualidade do Local de Trabalho, apesar de os enfermeiros de Internamento apresentarem menor satisfação em face dos órgãos de gestão, no que se refere ao estado de espírito enquadram-se na categoria de maior satisfação. Ou seja, apesar de se manifestarem menos satisfeitos com a comunicação, com a forma como se sentem reconhecidos e como as suas queixas e objeções são tratadas pelos órgãos de gestão, sentem-se satisfeitos por trabalharem nos seus contextos de prática clínica e quanto à forma como pensam ser percebida pelos outros por esse fato.

As caraterísticas do ambiente de trabalho, quer de natureza intrínseca (que decorrem da prestação direta de cuidados), quer extrínseca (onde se incluem as questões relacionadas com as políticas organizacionais, liderança e relações interpessoais, a que se refere a QLT), são mediadoras da satisfação profissional e, esta, por sua vez, da qualidade dos cuidados ${ }^{(14,19)}$. A par do que se verificou com a satisfação em face aos órgãos de gestão, a maioria dos enfermeiros apresenta uma menor satisfação com a QPC. A totalidade dos enfermeiros inquiridos de Cuidados Críticos, de Internamento, Ambulatório, das UCSP/USP e Cuidados Continuados apresenta-se menos satisfeita quanto à forma como é prestado o atendimento e como as informações aos usuários são fornecidas, à sensibilidade e forma como os profissionais desempenham as suas funções. A percepção de não prestação de cuidados de elevada qualidade é promotora da insatisfação profissional, podendo por si só conduzir e/ou agravar o decréscimo nessa mesma qualidade ${ }^{(20-21)}$. Os enfermeiros possuem concepções muito definidas e estruturadas dos padrões de qualidade, podendo contribuir para a elevação do seu grau de exigência, o que poderá contribuir para a compreensão da menor satisfação verificada entre estes enfermeiros $^{(22)}$. Ainda assim, destacaram-se dois grupos de enfermeiros com maior satisfação em face a esta dimensão: os das UCC e Cuidados Indiretos. Este dado poderá estar relacionado com a justificação relativa ao modelo organizacional, em que a autonomia e independência poderão ter contribuído para destacar a satisfação profissional dos enfermeiros das UCC com relação a esta dimensão, sendo esta uma caraterística da organização do trabalho similar à desenvolvida nos Cuidados Indiretos ${ }^{(23)}$.

Os enfermeiros com maior satisfação quanto à MCQ são os de Cuidados Indiretos e das UCC e os com menor são os de Cuidados Críticos e de Ambulatório. Apesar da identificação de menor satisfação face à QPC por cinco dos grupos de enfermeiros participantes, o mesmo não foi observado relativamente ao esforço desenvolvido pelas unidades para investir na qualidade dos cuidados de forma continuada.

\section{Grau de Satisfação por ÁRea de cuidados}

Os resultados quantitativos da análise da SP total são corroborados pelo grau de satisfação, sendo os enfermeiros que trabalham nas UCC os que se percebem mais satisfeitos e, por outro lado, os menos satisfeitos são os que trabalham nas unidades de Ambulatório e Cuidados Críticos. A menor satisfação dos enfermeiros de cuidados críticos é sustentada pela literatura ${ }^{(24)}$, sendo este o grupo de enfermeiros que apresenta menor satisfação quando comparado com as restantes áreas. Tal poderá dever-se à dificuldade no acesso a horários de trabalho flexíveis, o que contribui para o desequilíbrio do binômio trabalho-família, pois relata-se maior qualidade de vida profissional do que familiar ${ }^{(14)}$. Adicionalmente, a literatura identifica ainda a maior prevalência de níveis elevados de burnout e probabilidade de deixar a profissão entre os enfermeiros de cuidados críticos devido à exposição a ambientes de trabalho demasiado estressantes ${ }^{(25)}$.

\section{LIMITAÇÕES DO ESTUDO}

Identificou-se uma baixa taxa de resposta dos participantes da área de Cuidados Continuados, o que pode ter limitado a compreensão da variável satisfação profissional neste contexto específico. Nesse sentido, a existência de algumas amostras de tamanho reduzido poderá limitar parte da generalização dos resultados.

\section{CONCLUSÃO}

Os resultados do estudo possibilitaram verificar que a área de cuidados influencia a satisfação profissional dos enfermeiros. Identificou-se que os enfermeiros das Unidades de Cuidados na Comunidade e Cuidados Indiretos 
demonstram maior satisfação total em face ao restante de enfermeiros da ULS. A análise por dimensão possibilitou identificar que a satisfação profissional dos enfermeiros das UCC é globalmente mais elevada, com exceção das dimensões qualidade da prestação de cuidados e melhoria contínua da qualidade. Nestas últimas dimensões, os enfermeiros com maior satisfação são os de cuidados indiretos. Face à qualidade do local de trabalho, destacou-se a menor satisfação da maioria dos enfermeiros em relação aos Órgãos de Gestão.

Destacou-se, ainda, serem os enfermeiros de Cuidados Críticos os menos satisfeitos com todas as dimensões da satisfação profissional, o que merece uma atenção particular por parte dos responsáveis da ULS.

Em consonância com resultados anteriores, o estudo evidenciou que os próprios enfermeiros das UCC e de Cuidados Indiretos se reconhecem como mais satisfeitos, os de Cuidados Críticos menos satisfeitos e os das unidades de Ambulatório tendem para um ponto neutro.

Apesar de as áreas de Cuidados de Saúde Primários, Cuidados Hospitalares e Cuidados Continuados de uma ULS deverem ser encaradas como uma só organização, numa perspectiva holística do cuidar e da sua missão conjunta, em que o todo é mais que a soma das partes, do ponto de vista da gestão de recursos humanos a abordagem deverá ter em conta as especificidades dos contextos e a promoção de estratégias de satisfação profissional adequadas à avaliação realizada pelos seus profissionais.

Sugere-se a realização de uma análise independente da satisfação profissional em cada área para uma identificação mais fina da percepção dos enfermeiros, aproveitando as potencialidades proporcionadas pela coleta de dados já realizada.

\section{RESUMO}

Objetivo: Analisar o grau de satisfação profissional dos enfermeiros segundo a área de prestação de cuidados numa Unidade Local de Saúde, em Portugal. Método: Estudo quantitativo do tipo descritivo, analítico, com desenho observacional e transversal. Utilizou-se o Instrumento de Avaliação da Satisfação Profissional para a coleta de dados. Resultados: Amostra $(n=218)$, predominantemente feminina (84,6\%), com a idade média de 33 anos. A área de prestação de cuidados influencia a satisfação profissional total $(p<0,05)$ e algumas das dimensões estudadas $(p<0,05)$. Os enfermeiros das Unidades de Cuidados na Comunidade apresentam maior satisfação profissional, seguidos dos enfermeiros de Cuidados Indiretos. Os enfermeiros de Cuidados Críticos apresentam menor satisfação profissional, seguidos dos que trabalham em unidades de Ambulatório. A maioria dos enfermeiros apresenta menor satisfação na avaliação da faceta Orgãos de Gestão e na dimensão Qualidade da Prestação de Cuidados. Conclusão: Os enfermeiros mais satisfeitos são os das Unidades de Cuidados na Comunidade e os enfermeiros com menor nível de satisfação profissional são os de Cuidados Críticos.

\section{DESCRITORES}

Satisfação no Emprego; Enfermeiras e Enfermeiros; Assistência à Saúde.

\section{RESUMEN}

Objetivo: Analizar el grado de satisfacción profesional de los enfermeros según el área de atención en una Unidad Local de Salud, en Portugal. Método: Estudio cuantitativo de tipo descriptivo, analítico, con diseño observacional y transversal. Para la recopilación de datos se utilizó el Instrumento de Evaluación de la Satisfacción Profesional. Resultados: Muestra $(n=218)$, predominantemente femenina (84,6\%), con una edad promedio de 33 años. El área de prestación de cuidados influye en la satisfacción laboral total ( $\mathrm{p}<$ $0,05)$ y en algunas de las dimensiones estudiadas $(p<0,05)$. Los enfermeros de las Unidades de Cuidados de la Comunidad tienen mayor satisfacción laboral, seguidos de los enfermeros de Atención Indirecta. Los enfermeros de cuidados intensivos tienen menor satisfacción laboral, seguidos de los que trabajan en unidades ambulatorias. La mayoría de los enfermeros presentan menor satisfacción en la evaluación de la faceta Órganos de gestión y en la dimensión Calidad de la prestación de atención. Conclusión: Los enfermeros más satisfechos son los que trabajan en las Unidades de Cuidados de la Comunidad y los enfermeros con menor nivel de satisfacción profesional son los de Cuidados Intensivos.

\section{DESCRIPTORES}

Satisfacción en el Trabajo; Enfermeras y Enfermeros; Prestación de Atención de Salud.

\section{REFERÊNCIAS}

1. Silva CN, Potra TM. Job satisfaction of nurses in a local health unit: determinants of satisfaction]. Rev Enferm Ref. 2019;20:117-28. Portuguese. https://doi.org/10.12707/RIV18077

2. Marqueze EC, Moreno CR. Job satisfaction: a short review. Rev Bras Saúde Ocup. 2005;30(112):69-79. https://doi.org/10.1590/S030376572005000200007

3. Rego A, Cunha MP, Gomes JF, Cunha RC, Cabral-Cardoso C, Marques CA. Manual de gestão de pessoas e do capital humano. 3a ed. Lisboa: Sílabo; 2015.

4. Laschinger S, Heather K, Fida R. Linking Nurses' Perceptions of Patient Care Quality to Job Satisfaction. J Nurs Adm. 2015;45(5):276-83. https://doi.org/10.1097/NNA.0000000000000198

5. Portugal. Decreto-Lei № 238/2012. 2012. Procede à criação, com a natureza de entidade pública empresarial, da Unidade Local de Saúde do Litoral Alentejano, E. P. E., por integração do Hospital do Litoral Alentejano e do Agrupamento de Centros de Saúde do Alentejo Litoral. Diário República, 2012 out. 31: 6288-97.

6. Santana RU, Costa C. [Vertical integration : definition and organizational issues]. Rev Port Saude Publica. 2008;7:29-56. Portuguese. Available from: https://pdfs.semanticscholar.org/0997/6809564be0d4a6a3067f3f38d572e55b1831.pdf

7. Hill M, Hill A. Investigação por questionário. 2a ed. Lisboa: Sílabo; 2008.

8. Maroco J. Análise estatística: com utilização do SPSS. Lisboa: Sílabo; 2007. 
9. Moura S, Brás M, Anes E, Ferreira C. Satisfação no trabalho em profissionais de enfermagem. Bragança: Instituto Politécnico de Bragança; 2016. Available from: http://hdl.handle.net/10198/13231

10. Ferreira CA, Fernandez RF, Anes EM. [Job satisfaction of nurses working in hospital units of northern Portugal]. Rev Referência. 2017;4(15):109-20. Portuguese.https://doi.org/10.12707/RIV17043

11. Atefi N, Abdullah KL, Wong LP, Mazlom R. Factors influencing registered nurses perception of their overall job satisfaction: a qualitative study. Int Nurs Rev. 2014;61(3):352-60. https://doi.org/10.1111/inr.12112

12. Weston MJ. Strategies for enhancing autonomy and control over nursing practice. Online J Issues Nurs. 2010;15(1): https://doi.org/10.3912/ OJIN.Vol15No01Man02.

13. Poghosyan L, Liu J, Shang J, D'Aunno T. Practice environments and job satisfaction and turnover intentions of nurse practitioners: implications for primary care workforce capacity. Health Care Manage Rev. 2017;42(2):162-71. https://doi.org/10.1097/HMR.000000000000009414

14. Silva CN, Potra TS. [Job satisfaction of nurses: a scoping review]. Pensar Enferm [Internet]. 2017 [cited 2017 Oct 2];21(2)33-50. Available from: http://pensarenfermagem.esel.pt/files/Artigo3-Paginas33_50.pdf

15. Mendes AP. Satisfação profissional dos enfermeiros de cuidados de saúde primários do ACES Baixo Mondego II (Dissertation). Coimbra: Escola Superior de Enfermagem de Coimbra; 2014.

16. M Alshahrani FM, Baig LA. Effect of leadership styles on job satisfaction among critical care nurses in Aseer, Saudi Arabia. J Coll Physicians Surg Pak. 2016 [cited 2008 Aug 2];26(5):366-70. Available from: https://www.researchgate.net/publication/319665381_Effect_of_ Leadership_Styles_on_Job_Satisfaction_Among_Critical_Care_Nurses_in_Aseer_Saudi_Arabia

17. Cunha MP, Cunha RC, Rego A, Neves P, Cabral-Cardoso C. Manual de comportamento organizacional e gestão. 8a ed. Lisboa: Editora $\mathrm{RH} ; 2016$.

18. Platis C, Reklitis P, Zimeras S. Relation between job satisfaction and job performance in healthcare services. Procedia Soc Behav Sci. 2015;175:480-7. https://doi.org/10.1016/j.sbspro.2015.01.1226

19. Bai J. Does job satisfaction mediate the relationship between healthy work environment and care quality? Nurs Crit Care. 2016;21(1):1827. https://doi.org/10.1111/nicc.12122

20. Lu M, Ruan H, Xing W, Hu Y. Nurse burnout in China: a questionnaire survey on staffing, job satisfaction, and quality of care. J Nurs Manag. 2015 May;23(4):440-7. https://doi.org/10.1111/jonm.12150

21. Shin JI, Lee E. The effect of social capital on job satisfaction and quality of care among hospital nurses in South Korea. J Nurs Manag. 2016 Oct;24(7):934-42. https://doi.org/10.1111/jonm.12401

22. Hayes B, Bonner A, Pryor J. Factors contributing to nurse job satisfaction in the acute hospital setting: a review of recent literature. J Nurs Manag. 2010 Oct;18(7):804-14. https://doi.org/10.1111/j.1365-2834.2010.01131.x

23. Dominique-Ferreira S. [Measuring the satisfaction of health professionals: an application on the Health Centers Group of Feira-Arouca]. Rev Port Saude Publica. 2015;3(2):188-98. Portuguese. https://doi.org/10.1016/j.rpsp.2015.07.001

24. Ylitörmänen T, Turunen $\mathrm{H}$, Kvist T. Job satisfaction among registered nurses in two Scandinavian acute care hospitals. J Nurs Manag. 2018;26(7):888-97. https://doi.org/10.1111/jonm.12620 PMID:30136430

25. Alharbi J, Wilson R, Woods C, Usher K. The factors influencing burnout and job satisfaction among critical care nurses: a study of Saudi critical care nurses. J Nurs Manag. 2016;24(6):708-17. https://doi.org/10.1111/jonm.12386

Unidade de Investigação e Desenvolvimento em Enfermagem (UI\&DE). 